\title{
EL ABOLENGO GITANO-INDIO DE CHAVÓ Y SU FAMILIA
}

En esta revista, XI (1956), 229-234, el señor José Vázquez Ruiz sostiene que, a su juicio, la voz chaval y sus derivados tienen su étimo en la lengua árabe.

Tratándose de una palabra de no dudoso origen andaluz y gitano, la primera pregunta que uno debe hacerse es si esta palabra pertenece al solo gitano español o si es común a otros dialectos gitanos. Esta pregunta no se la pone ni siquiera el señor Vázquez Ruiz.

Ahora bien, la palabra chavó "niño, muchacho» es de pura cepa gitana y está difundida en todos los dialectos gitanos europeos desde Turquia a través de Europa hasta Inglaterra y España, como ya observé en mi Argot barcelonais, p. 103. Referencias exhaustivas se encuentran en las obras de A. F. Pott, Die Zigeuner in Europa und Asien, Halle I845, vol. II, p. I8I y de F. Miklosich, Ueber die Mundarten und Wanderungen der Zigeuner Europas, I2 vols. (Viena, I872-I880, vol. VII, página 30). Tagliavini, en C. Tagliavini-A. Menarini, Voci zingare nel gergo bolognese, Florencia, I938 ( = AR, XXII (I938), 242-280), pp. 253 y siguientes, ha recogido todas las formas encontradas en los diccionarios dialectales gitanos publicados después de la fecha de la salida de las obras de Pott y de Miklosich, y esta lista se podría todavia acrecentar con las indicaciones de otros trabajos aparecidos entre tanto.

Pero la misma palabra existe no solamente en los dialectos gitanos europeos, sino también en los de Asia.

En efecto, resulta de la obra de M. J. de Goeje, Mémoire sur les migrations des Tsiganes à travers l'A sie, Leide, r903, p. 4I, que:

I) en el dialecto de los gitanos de Egipto se dice $c h a v o$ o $c h a b o$, "garçon" (según T. J. Newbold, The Gypsies of Egypt, in Journal of the 
Royal Asiatic Society of Great Britain and Ireland, XVI (I856), 285-3r3, página 299),

2) en el dialecto de los gitanos de Persia: d j a v a ugarçon* (según A. de Gobineau, Persische Studien, en ZDMG, XI (1857), 689-707 página 695),

3) en el dialecto de los gitanos de Siria: $t s c h \bar{a} g a, c h a ̈ g h a$ o chogo, según varios autores.

Pott y Miklosich no se decidieron por ninguna etimologia, y por esto, yo también observé en mi Argot barc., p. I03, "d'origine incertaine", a lo que se refiere el señor Vázquez Ruiz, p. 232 (García de Diego, en su Diccionario etimológico español e hispánico, p. 207, dice también de $c h a v a l$ "de origen incierto»).

Pero estudios más recientes no admiten dudas sobre el origen indio de la palabra. John Sampson, en su monumental obra The dialects of the Gypsies of Wales, Oxford, I926, p. 55, señala como correspondencias indias de nuestra palabra el sánscr. $s \bar{a} v a^{2}$, pāli $c h \bar{a} p a$, prikr. $c h \bar{a} v a$ uthe young of an animal», formas que ya J. Beames, Comparative grammar of the modern Aryan languages of India, Londres, 18721879, p. 26I. y Rich. Pischel, Grammatik der Präkrit-Sprachen, Estrasburgo, I900, § 2II, habian equiparado con las palabras gitanas. Compárese, además, nepali $c h \bar{a} w \bar{a}$ "young of an elephant or camel» in Ralph Lilley Turner, $A$ comparative and etymological dictionary of the Nepali language, Londres, I93r, p. I96.

El que el significado originario de "cría de animal» se haya aplicado a un chiquillo no tiene nada de extraño; compárese el inglés $k i d$, originariamente "cabrito», usado hoy en día generalmente por «niño, chica» (sobre todo en América), y las numerosas metáforas sacadas del reino animal para denominar niños en Ivan Pauli, Enfant, garçon, fille dans les langues romanes, Lund, I9I9, pp. 297 y sigs.

El señor Vázquez Ruiz se ha fijado demasiado en la forma cha $v a l$. sin tener en cuenta las otras formas. Ahora bien, la forma básica de toda la familia es chavó, no chaval.

El artículo chaval del Dicc. de Corominas (II, p. 39), al que el autor se refiere, reproduce lo apuntado por mí en Stray Notes on Spanish Romani, en Journal of the Gypsy Lore Society, 3 rd ser., vol. XVI (I937). p. 32, 10 que dice expresamente Corominas.

1 La correspondencia präkr. cha-: sánscr. $s a-$, que puede parecer extraña a un no-iniciado, es completamente normal, véase Pischel, l. c., p. 152, quien dice a propósito: Anlautender einfacher Zischlaut des Skt. wird im Prākr. zuweilen aspiriert: $s h a, s h a, s h a$ werden dann gleichmässig zu cha* . 
En aquel artículo traté de explicar las distintas formas del gitano español:

ch $a v \delta$ que, como hemos visto, es una forma difundida en todos los dialectos gitanos, es el nom. masc. sing. regular;

$c h a v \dot{E}$, que en el gitano esp. actual se usa también como un nominativo sing., es originariamente la forma del nom. plur., asimismo regular. Ya que en el git. esp. la noción del valor funcional de los casos gitanos se ha borrado, los plurales originarios se emplean a menudo como singulares, y de la misma manera se dice $g a c h \delta$ y $g a c h e ́$ qhombre no gitanor; c a lo y calé, etc. (el plural de todos los substantivos se forma según el modelo español: $c h a v o ́ s$ o $c h a v e ́ s, g a-$ chos, gachés, etc.).

chaval es el originario vocativo plural: chavale, que como tal vive todavía en los dialectos que han conservado la antigua flexión (Sampson, l. c.; git. alemán: ts $\bar{a} w a l e$ "Jungens" (F. N. Finck, Lehrbuch des Dialekts der deutschen Zigeuner, Marburg, I903, p. I7).

c h a ve a "chiquillo", usado en el esp. git. y en andaluz familiar (Toro y Gisbert, Voces andaluzas, p. 412; Alcalá Venceslada ${ }^{2}$, p. I96), se deriva del vocativo sing. chaváia (vivo aún en los dialectos gitanos más puros): Sampson, l. c.; git. alemán: $t \xi a$ wé $j a$ : Finck, l. c., p. 20;

$c h a i$ uniña, mocita» es una contracción de $c h a v i$, que ya pertenece al gitano originario, p. ej., git. turco: $t s c h a \dot{i}, t s c h e ́ i$ (A. G. Paspati, Etude sur les Tschingianés ou Bohémiens de l'Empire Ottoman, Constantinopla, I87o, s. v); gitano galés: $c h a i$ (Sampson, p. 55);

chavoró, chaboró «hijo» es un originario diminutivo regular (gitano turco: $t s c h a v o r o ́:$ Paspati); git. galés: chavoró (Sampson, p. 56); cfr., además, Pott, II, I82; Miklosich, VII, 30.

En los dialectos indios -ora es el sufijo ordinario para formar diminutivos; se continúa como oró en los dialectos gitanos orientales de Turquia, Rumania y Hungria, donde el valor diminutivo del sufijo está todavía vivo y donde este sufijo puede añadirse a cualquier substantivo. Pero en el git. galés y en casi todos los dialectos gitanos del norte de Europa ha cesado de ser un sufijo vivo y subsiste tan sólo en algunas palabras tradicionales, entre las cuales chavoro "little boy" (Sampson, parte I. ${ }^{\mathrm{a}}, \S$ I55).

Igual sucede en el git. esp. que ha perdido completamentela noción del valor funcional de este sufijo, pero también en git. esp. hay algunos restos fosilizados de -oró en palabras como chavoró; plaloró "hermano" al lado de $p l a l$ uid.".

Como llevamos dicho, ya no hay en el git. esp. sino pocos vestigios de la antigua flexión. Se hace todavía la distinción entre el masc. en 
- $\delta$ o - $\&$ y el fem. en $-\boldsymbol{f}$. Todos los otros casos de la antigua declinación han desaparecido o viven solamente en formaciones fosilizadas ya no comprendidas. Por lo tanto, todas las variantes mencionadas: $c h a v \delta$, chavé, chaval, chavea, chavoró se usan hoy como nominativos, y más o menos con el mismo valor.

Esta confusión explica cómo en el caló catalán $x a$ v e i $a$ se ha - vuelto fem. e indica uniña», gracias al - a final (Argot barc., p. ro3), y es natural que haya además los femeninos $x a v a$ en el mismo caló barcelonés, y chavala en España, y que se forman nuevos diminutivos: $c h a v a l i t o$; cat. $x i b a l$ ét unoyet, mozuelo" (ib.).

En mi citado artículo Stray Notes traté de estos casos en el capítulo titulado Flexional confusion (XVI, pp. 30-32). Un ejemplo muy instructivo es $b u l l a t e$, que se usa al lado de $b u l$ utrasero" ( $b u l l a t i$ whe anus" en Borrow, y $b u l l$ a te en Rebolledo, p. 26; g u y a $t e$ en la novela andaluza Hampa y Miseria, de José Más, p. 46, donde un gitano dice a un muchacho: $-A$ cayá, mardecido, o te cierro pa siempre de una patá er cliso der gu y a te»).

En git. esp. $b u l$ es hoy de género masc., pero en los dialectos genuinos es fem. (Sampson, p. 47; sánscr. $b u$ l.i «vulva», kanus»). Pero $b u$ l l ate, con la terminación del ablativo de los substantivos femeninos: - i at $e(r)$ : Pott I, 186; Miklosich, XI, 2; Sampson, § 295 (el ablativo masc. se termina en - $e ́ s t e$ ) prueba que antiguamente la palabra debió ser fem. también en España ( $y$, efectivamente, se dice todavía en el caló catalán: no val una bul (Givanel i Mas, $B D C$, VII, 25; Argot barc., 42). Ahora bien, este ablativo es hoy también nominativo, y el que' haya sobrevivido, aunque fosilizado, se debe probablemente a su uso frecuente en ciertas imprecaciones gitanas, como mo $\dot{k}$ a $r$ lés kere buliate umon pudendum dans son derrière" (Paspati, p. 267, s. v. (ar); te továv les léskere vuliate uà le mettre dans son derrière (sodomie)": (ib., p. 583 ).

Un caso parecido es el de mollate, que se usa en git. esp. equivalente a $m$ o $l$ uvino»; $m$ o $l l$ a $t e$ ha pasado al lenguaje popular español en el sentido de "vino tinto», y como tal está registrado por el $\mathrm{Pe}$ queño Larousse, por Besses, Dicc. de Argot español, p. 109, por el Diccionario español-alemán de Slabý-Grossmann, por Alcalá Venceslada", página 409 ("vino corriente»).

m ol uvino" ocurre en todos los dialectos gitanos (Pott, II, 455; Miklosich, VIII, r8; Sampson, p. 225) y es de origen indio. En el git. español es de género masc., pero en todos los dialectos genuinos es fem., y por esto su ablativo es mo li a $t e$. Se repite el fenómeno que hemos observado en $b u l, b u l l a t e, y$ por las mismas causas, es decir, 
por imitación del género de las correspondientes palabras españolas (culo, vino).

Para volver a $c h a v o ́$, su origen gitano-indio, en vista de los antecedentes y la difusión de la voz y de su familia, no deja lugar a dudas, y es casi de sobra detenerse a confutar la etimología árabe del señor Vázquez Ruiz.

Aun desde el punto de vista fonético la derivación de chaval del árabe $x e b e b$ "joven» chocaria con bastantes dificultades. El autor, es verdad, dice (p. 23I): "el primer miembro silábico $x e$ - procedente del árabe $\xi e>\xi a$ es normaln, remitiendo a Steiger, Contribución. p. I95 sig. Pero en el respetivo capítulo Steiger trata del árabe, \& $\xi$-, y no de $\gtrless^{\mathscr{Z}-,}$ y $\xi_{-}$(pp. I9.5 y sigs.) da regularmente en esp. asimismo $\zeta$-. No hay ni un solo ejemplo de árabe $\zeta$-: esp. ch-.

El señor Vázquez Ruiz sostiene que $s$ e $b$ e $b$ habría dado $x e b e l$ $>x a b a l>c h a v a l, y$ cita, para justificar este desarrollo, el portugués ant. al motacel<muhteceb (Steiger, p. Iro), y el español acëbile< ZIBIBE unvas pasas» (Steiger, p. I08).

E1 último ejemplo debe desecharse desde luego, porque es una forma inexistente y evidente errata en vez de $a c e b i b e$, como resulta también de Steiger, p. 343 y del índice de palabras, p. 464 , donde sólo se cita la forma exacta $a c e b i b e$.

Queda el port. ant. a $l$ m ot a $c$ e $l$. Pero éste es un caso completamente aislado que no se puede considerar como normal y que exige una explicación aparte.

Las palabras árabes con $-b(b)$ dan regularmente formas con - $b$ en español y port. también: Algunas veces el $-b$ se vuelve - $f$, lo que se comprende, desde luego. Entre los ejemplos de Steiger, p. Io9 y sig., hay uno sólo con $-l$, y es precisamente el port. ant. a $l m$ o $t a c e l$, forma secundaria de a lmotacé. La forma a lmotacel se explica por lo visto partiendo de la otra: a $l m$ o $t$ a $c \dot{e}$. No es raro el caso en que palabras con una vocal final transforman ésta en $-a l,-\dot{e} l,-i l$, etc. (cfr. el muy difundido dial. ja $b$ aril "jabalí»; port. $l a m b r i l=$ franc. $l a m-$ $b r i s$; andal. y amer. $b i d e l=b i d \dot{e}$, etc.; otros ejemplos en mi artículo sobre $m a r g u a l$ en $R F E$, XXI, 1934, pp. 237-243.

De todos modos, este ejemplo no tiene ningún valor y no permite establecer una regla, según la cual un árabe, $-b$, se haria $-l$ en palabras españolas y portuguesas.

El señor Vázquez Ruiz hasta nos quiere enseñar que las formas $c h a v e a$ y $c h a v o ́$ sean idénticas a $c h a v a l$ : "Las voces $c h a v e a$ y $c h a v o ́$ se originarían de la misma palabra, como consecuencia de un fenómeno de disimilación en el complejo final: $x e b \check{e} b>x e b \ddot{e} u$ 
$>x e b e a>c h a v e a, y x e b e b>x e b e r u>x e b \delta>c h a v b$. Para el presunto primer fenómeno el autor se remite al libro de Steiger, páginas ro7-ro8. Pero Steiger dice sólo de la -b-intervocálica que a veces se vocaliza en $u$, mientras de la $-b$ final observa: «Se reproduce siempre por $b$, en cat. y sic. a veces por $p_{m}$. Por consiguiente, no se puede mantener tampoco la segunda afirmación del autor.

Los desarrollos fonéticos supuestos por el autor no corresponden a la realidad.

En pág. 233 dice el autor, aludiendo a un pasaje del libro de Carlos Claverfa, Estudio sobre los gitanismos del español, Madrid, I951, p. I39: donde éste dice que $c h a v \delta$ ha adquirido asimismo, a través del vocativo, valor de interjección" lo siguiente: "Su función vocativa e interjectiva, de la que se ocupa Clavería, fué, sin duda, heredada o, mejor, copiada por los gitanos que hemos visto se establecieron en Granada y mantuvieron un trato constante con los moriscos». E1 autor se refiere al uso árabe de $y a \xi{ } b a b$. Pero él ha entendido mal las observariones de Claveria. Que $c h a v \delta$ pueda usarse como vocativo, es cosa notural, pero esto no tiene nada que ver con el vocativo árabe (el árabe nn posee un caso especial para el vocativo, sino lo hace preceder de y $a$, inmo el latino y otras lenguas se sirven de $o$ ). Para el gitano es cosa d stinta: chaval y chavea eran originariamente vocativos, pero han perdido completamente este valor funcional en el gitano español, lo que naturalmente no impide que $c h a v a l$ y $c h$ a $v$ e a puedan usarse también como vocativos de la misma manera que $c h a v \delta$ y como un nombre de persona cualquiera. Pero esto no tiene ninguna conexión con su valor originario, ni con el uso árabe.

En resumidas cuentas, se trata de una voz de indudable origen gitano-indio que hasta conserva, en formas fosilizadas, algunos casos de la antigua flexión y el antiguo modo de formar los diminutivos.

La etimología árabe del señor Vázquez Ruiz, por eruditas que parezcan sus ilaciones, no se puede aceptar de ninguna manera.

† M. L. WAGNER 